\title{
The Order Parameter in a Spin Glass
}

\author{
A. C. D. van Enter ${ }^{1}$ and Robert B. Griffiths ${ }^{2}$ \\ I.H.E.S., 35 route de Chartres, F-91440 Bures-sur-Yvette, France
}

\begin{abstract}
Various possible precise definitions of an Edwards-Anderson type of order parameter for an Ising model spin glass are considered, using boundary conditions for a finite system, states of an infinite system, and a duplicate-system approach. Several of these definitions are shown to yield identical results.
\end{abstract}

\section{Introduction}

It is generally believed that if spin glass ordering can be described within the framework of equilibrium statistical mechanics, it corresponds to a non-zero value of the Edwards-Anderson order parameter [1]

$$
q_{E A}=\left\langle\left\langle S_{i}\right\rangle^{2}\right\rangle
$$

where $S_{i}$ is the magnetization at site $i$, the inner brackets refer to a thermal average, and the outer brackets an average over an ensemble of random systems. As it stands, this definition is ambiguous, since it will depend, in general, on the choice of boundary conditions for finite random systems.

For a random Ising model (Sect. II) we consider some alternative definitions (Sect. III) which have the virtue of being well-defined in the thermodynamic limit. One is a "thermodynamic" definition [2], one is based on states of an infinite system, and one on the maximization of a quantity like (1) over all possible boundary conditions. We show (Sect. IV) that in the thermodynamic limit they are all welldefined and equal to each other. For simplicity of exposition, the discussion is limited to zero magnetic field, but its extension to non-zero field (Sect. V) causes no difficulty. Yet another rather appealing definition (Sect. VI) encounters some technical difficulties we have not resolved.

\section{Spin-Glass Model}

For simplicity of exposition and in order to avoid having to state a host of technical restrictions, we limit our discussion to a random Ising model $S_{i}= \pm 1$ with

1 Present address: Universität Heidelberg, Sonderforschungsbereich 123, 6900 Heidelberg 1, Federal Republic of Germany

2 Permanent address: Physics Department, Carnegie-Mellon University, Pittsburgh, PA 15213, USA 
dimensionless Hamiltonian (i.e., the energy divided by $-k T$ ) equal to

$$
H(\tau)=\frac{1}{2} \sum_{i j} J(i-j) \tau_{i j} S_{i} S_{j}+B \sum_{i} S_{i}
$$

Here $i$ and $j$ are vector locations of sites on a Bravais lattice of arbitrary (but finite) dimensionality $d, B$ is a magnetic field, $J(i-j)=J(j-i)$ is an interaction of finite range depending only on the vector connecting the sites, and for each pair $i, j$ with $J(i-j) \neq 0, \tau_{i j}=\tau_{j i}$ is a real random variable. The $\tau_{i j}$, denoted collectively by $\tau$, are independently distributed; for each pair $i, j$ the distribution has a finite first moment, and depends only on $i-j$.

The Hamiltonian $H_{\Lambda}$ for a finite set $\Lambda$ containing $|\Lambda|$ lattice sites is obtained by restricting the sums in (2) to $i$ and $j$ in $\Lambda$. The corresponding dimensionless free energy for the finite system, $f_{\Lambda}(\tau)$, is given by

$$
f_{\Lambda}(\tau)=|\Lambda|^{-1} \ln \operatorname{Tr}_{\Lambda}\left[\exp H_{\Lambda}(\tau)\right]
$$

where $\operatorname{Tr}_{\Lambda}$ is the sum over the $S_{i}$ with $i \in \Lambda$. This can be averaged over $\tau$ to yield

$$
\bar{f}_{\Lambda}=\int f_{\Lambda}(\tau) \mathrm{d} \mu(\tau)
$$

where $\mu$ is the probability measure for $\tau$. It can be shown that as $\Lambda \rightarrow \infty$ in the sense of van Hove, $\bar{f}_{A}$ approaches a unique limit $f[3]$, and that with probability one $f_{\Lambda}(\tau)$ approaches the same limit as $\Lambda \rightarrow \infty[4,5,6]$. In what follows we shall restrict ourselves to regions $\Lambda$ which are rectangular boxes. A sequence of such boxes which eventually contains every site of the lattice is then a van Hove sequence.

\section{Order Parameters}

For a given $\tau$, that is to say, a set of $\tau_{i j}$, there will in general be a number of equilibrium states for the infinite system satisfying the DLR equations [7] and (equivalently) certain variational properties [8]. In the case of non-random systems with interactions invariant under translations (as in the usual Ising ferromagnet), the translationally invariant equilibrium states are often thought of as pure phases. However, non-translationally invariant states are also possible (as in antiferromagnets, or the states describing interfaces in Ising ferromagnets with $d \geqq 3$ ). From this point of view, "phase coexistence" or "ordering" corresponds to the existence of more than one equilibrium state, and an order parameter should in some sense be a measure of the extent by which different equilibrium states differ from each other.

In what follows, we shall, to simplify the exposition, consider only the case $B=0$, and at the end of the paper indicate the appropriate modifications for $B \neq 0$. Our first proposal for our order parameter corresponding to (1) is then

$$
q=\lim _{\Lambda \rightarrow \infty} \max _{\alpha}|\Lambda|^{-1} \sum_{i \in \Lambda}\left[\rho_{\alpha}\left(S_{i}\right)\right]^{2},
$$

where $\rho_{\alpha}(A)$ is the average of $A$ in the equilibrium state labeled by $\alpha$. The set of equilibrium states will, of course, depend upon $\tau$, so that the above definition will, in general, yield a $q$ which depends on $\tau$. However, as the subsequent argument will 
demonstrate, with probability $1, q$ does not depend on $\tau$, nor on the choice of the van Hove sequence. Of course the state $\alpha$ which leads to a maximum (there is always at least one such state, for the set of equilibrium states is compact in the weak-* topology) could very well depend upon $A$, and the reader may well wonder whether the maximum (or supremum) over $\alpha$ could not be taken after the thermodynamic $\operatorname{limit} \Lambda \rightarrow \infty$. We comment on this point at the end of the paper. One further remark about this definition: if the equilibrium state is unique (as, for example, one expects it to be at high temperatures) then $\rho_{\alpha}\left(S_{i}\right)$ is zero by the spin reversal symmetry $\left(S_{i}\right.$ replaced by $-S_{i}$ for all $i$ ) of $H$ when $B=0$, and hence $q=0$.

The preceding definition makes use of states of the infinite system, but for a given $\Lambda$ only makes use of the properties of these states restricted to sites in $\Lambda$. A closely related definition is as follows. For a given finite $\Lambda$, let $\bar{\Lambda}$ be the sites of $\Lambda$ together with all sites $j$ outside $\Lambda$ such that $J(i-j) \neq 0$ for some site $i$ in $\Lambda$. As the interactions have finite range, $\bar{\Lambda}$ will be finite. Let $\bar{\rho}_{\Lambda, \mu}$ be the Gibbs distribution for the $S_{i}, i \in \Lambda$, given that the "boundary spins" $S_{j}, j$ in $\bar{\Lambda} \backslash \Lambda$, denoted collectively by $\mu$, have specified values:

$$
\bar{\rho}_{\Lambda, \mu}=\left(\exp H_{\bar{A}}\right) / \operatorname{Tr}_{\Lambda}\left(\exp H_{\bar{A}}\right)
$$

where note that the denominator is a sum over $S_{i}$ with $i$ in $\Lambda$, not $\bar{\Lambda}$.

We now define

$$
\bar{q}=\lim _{\Lambda \rightarrow \infty} \max _{\mu}|\Lambda|^{-1} \sum_{i \in \Lambda}\left[\bar{\rho}_{\Lambda, \mu}\left(S_{i}\right)\right]^{2},
$$

where the maximization is now over all possible choices of spin variables in the boundary of $\Lambda$. Once again, the definition depends on $\tau$, but, as in the case of $q$, we shall show that with probability 1 a unique value is obtained, independent of the van Hove sequence, which is equal to $q$.

The thermodynamic definition ${ }^{1}$ is as follows. We construct two identical systems with the same $\tau$ and a coupling between corresponding sites so that the total Hamiltonian is

$$
H^{t}(\lambda, \tau)=H^{(1)}(\tau) \otimes 1+1 \otimes H^{(2)}(\tau)+\lambda \sum_{i} S_{i}^{(1)} S_{t}^{(2)},
$$

with $H^{(l)}$ obtained by replacing $S_{l}$ and $S_{j}$ in (2) by $S_{l}^{(l)}$ and $S_{j}^{(l)}$. We may think of the duplicates as lying on top of each other, so that any finite box $\Lambda$ contains the same set of sites for each. Replacing $H_{A}$ by $H_{A}^{t}$ in (3) and dividing the right side by 2 yields an $f_{\Lambda}(\lambda, \tau)$ which is identical to the previous $f_{\Lambda}(\tau)$ when $\lambda=0$, and has a thermodynamic limit (with probability 1) $f(\lambda)$ equal to $f$ when $\lambda=0$.

We now define the order parameter $q_{p}$ by

$$
q_{p}=2 f^{\prime}(0+)
$$

where $f^{\prime}$ denotes $d f / d \lambda$, and $0+$ the limit of this derivative as $\lambda$ goes to zero through positive values. As $f(\lambda)$ is convex, this limit exists, and is independent of $\tau$ with probability 1.

1 This order parameter, with a clear statement of the necessary limits, is defined in Eq. (17) of ref. [2] where it is ascribed to [17]. Similar ideas will be found in [18], Eq. (11b); [19], Eq. (B8); and [20] 


\section{Equality of Order Parameters}

We shall now show that $q, \bar{q}$, and $q_{p}$ are all equal. The first step is to establish that

$$
q \leqq \bar{q} \leqq q_{p}
$$

The first inequality follows from the fact that an equilibrium state of an infinite system restricted to a finite set $\Lambda$, as used in (5), is a convex combination of the $\bar{\rho}_{\Lambda, \mu}$, and hence the right side of (5) for a given $\Lambda$ (and $\tau$ ) cannot exceed the right side of (7) for the same $\Lambda$ (and $\tau$ ) [7]. The second inequality is the result of a very general argument by which a thermodynamic derivative in a classical system (commuting variables) places an upper bound on a probability distribution as $\Lambda \rightarrow \infty$ [9]. If the magnetization in ref. [9] (note that the free energy employed in that paper is concave) is replaced by $\sum S_{i}^{(1)} S_{i}^{(2)}$ in the duplicate system, and for a finite $\Lambda$ each of the duplicate systems has the same boundary condition, namely the one maximizing the right side of (7), the argument in [9] can be applied to show that $\bar{q} \leqq q_{p}$.

The second and less trivial step is to show that

$$
q_{p} \leqq q .
$$

To prove this, we introduce the following notation. For $\rho$ any state of the duplicate system, let

$$
R_{\Lambda}(\rho)=|\Lambda|^{-1} \sum_{i \in \Lambda} \rho\left(S_{i}^{(1)} S_{i}^{(2)}\right)
$$

and for a given $\lambda$ and $\tau$ let

$$
Q_{\Lambda}(\lambda, \tau)=\max _{\rho(\lambda, \tau)} R_{\Lambda}(\rho(\lambda, \tau))
$$

where $\rho(\lambda, \tau)$ is any equilibrium state corresponding to $H^{t}(\lambda, \tau)$. The following result is proved in App. $A$ :

Lemma. There is an upper semi-continuous function $q(\lambda)$ such that

$$
q(\lambda)=\lim _{\Lambda \rightarrow \infty} \int Q_{\Lambda}(\lambda, \tau) d \mu(\tau)
$$

for every van Hove sequence of rectangular boxes $\Lambda$. For a fixed $\Lambda$ the integral is not less than $q(\lambda)$. In addition, for a given $\lambda$ it is the case that for almost all $\tau$,

$$
q(\lambda)=\lim _{\Lambda \rightarrow \infty} Q_{\Lambda}(\lambda, \tau)
$$

for any van Hove sequence.

The quantity $q(0)$ is identical with $q$ as defined in (5). The reason is that when $\lambda=0$, the extremal equilibrium states of the duplicate system are products $\rho_{\alpha} \otimes \rho_{\beta}$ of equilibrium states of the two non-interacting pieces. Thus the lemma implies that for almost all $\tau$ the limit in (5) exists and is independent of the van Hove sequence.

To prove $(11)$, we use the variational principle $[8,11]$ which states that if $\rho$ is any state of the infinite system with Hamiltonian $H$,

$$
f_{\Lambda} \geqq F_{\Lambda}(\rho, H)=|\Lambda|^{-1}\left[S_{\Lambda}(\rho)+\rho\left(H_{\Lambda}\right)\right],
$$


where $f_{A}$ is defined by (3) and $F_{A}$ is the free energy per site calculated in the state $\rho$ restricted to $\Lambda$. In addition, if $\rho$ is an equilibrium state for $H$,

$$
f_{\Lambda} \leqq F_{\Lambda}(\rho, H)+2\left\|W_{\Lambda}\right\| /|\Lambda|
$$

(see the appendix of [10]), where $W_{A}$ is the sum of all terms in $H$ connecting the sites inside $\Lambda$ with the sites outside, and $\left\|W_{\Lambda}\right\|$ is the maximum value of this quantity. Although it is not noted explicitly in these equations, the reader should remember that $H, W_{\Lambda}, f_{\Lambda}$, and the equilibrium states depend on $\tau$.

Now let us assume that $\rho(\lambda, \tau)$ is an equilibrium state for the duplicate system with $\lambda>0$. Inserting it in (17) with $H$ equal to $H^{t}(\lambda, \tau)$ and in $(16)^{2}$ with $H$ equal to $H^{t}(0, \tau)$, and subtracting one equation from the other, we obtain

$$
\lambda^{-1}\left[f_{\Lambda}(\lambda, \tau)-f_{\Lambda}(0, \tau)\right] \leqq R_{\Lambda}(\rho(\lambda, \tau))+2\left\|W_{\Lambda}\right\| /|\Lambda| .
$$

As this inequality holds for any $\rho(\lambda, \tau)$, we can replace $R_{\Lambda}$ by $Q_{\Lambda}(\lambda, \tau)$. Upon integrating both sides over the probability distribution $\mu(\tau)$ and taking the limit $\Lambda$ $\rightarrow \infty$, we obtain

$$
q_{p}=f^{\prime}(0+) \leqq \lambda^{-1}[f(\lambda)-f(0)] \leqq q(\lambda),
$$

where the first inequality follows from the convexity of $f(\lambda)$. We now take the limit as $\lambda$ goes to zero through positive values and note that $q(\lambda)$ is upper semi-continuous in order to get (11). This completes the proof. (Note that the fact that $\bar{q}$ is "squeezed" between $q$ and $q_{p}$ implies that (7) cannot depend on the van Hove sequence.)

\section{Non-zero Magnetic Field}

When the external magnetic field $B$ is not zero,(5), (7), and (9) define quantities which will in general not be zero even when the equilibrium state is unique. To obtain an order parameter which is zero when the equilibrium state is unique, one can define

$$
q=\frac{1}{2}\left(q_{+}-q_{-}\right)
$$

where (5) is now the definition of $q_{+}$, and

$$
q_{-}=\lim _{\Lambda \rightarrow \infty} \min |\Lambda|^{-1} \sum_{i \in \Lambda} \rho_{\alpha}\left(S_{i}\right) \rho_{\beta}\left(S_{i}\right) \text {. }
$$

Similarly, $\bar{q}$ is defined in analogy with (20), letting (7) define $\bar{q}_{+}$and letting

$$
\bar{q}_{-}=\lim _{\Lambda \rightarrow \infty} \min |\Lambda|^{-1} \sum_{i \in \Lambda} \bar{\rho}_{\Lambda, \mu}\left(S_{i}\right) \bar{\rho}_{\Lambda, v}\left(S_{i}\right)
$$

Finally, the new definition of $q_{p}$ is

$$
q_{p}=\left[f^{\prime}\left(0_{+}\right)-f^{\prime}\left(0_{-}\right)\right] .
$$

The method employed above can then be used to show that $q, \bar{q}$, and $q_{p}$ are again equal; in fact, the " + " contributions are equal to each other, and likewise the " - " contributions are equal. 


\section{An Alternative Definition of the Order Parameter}

Finally, let us comment on interchanging the maximum with the thermodynamic limit in (5). It would certainly be appealing to define (for $B=0$; analogous considerations apply with $B \neq 0$ ) a quantity

$$
\hat{q}=\max _{\alpha} \lim _{\Lambda \rightarrow \infty}|\Lambda|^{-1} \sum_{i \in \Lambda}\left[\rho_{\alpha}\left(S_{i}\right)\right]^{2}
$$

as the order parameter, especially if one could show that $\hat{q}$ were equal to $q$. Obviously $\hat{q}$ cannot be larger than $q$, but one can imagine a state of affairs in which it would actually be smaller. Suppose there were an infinite number of equilibrium states, and that for each of these $R_{A}$ were to approach $\hat{q}$, or a smaller number, as $\Lambda \rightarrow \infty$. It could still be the case that for any finite $\Lambda$ one could choose a particular state giving an $R_{A}$ significantly larger than $\hat{q}$, provided the equilibrium states employed in (5) and (24) were inhomogeneous on arbitrarily large length scales. It seems difficult to rule out this possibility a priori.

In addition, the definition (24) suffers from a host of technical difficulties. The thermodynamic limit could fail to exist, or it could depend on the van Hove sequence considered. This difficulty can be "cured" by the somewhat awkward device of taking the lim sup for each van Hove sequence and the supremum of these over all van Hove sequences (compare [12]). The resulting limit may or may not be a continuous function of the state $\alpha$ in the weak-* topology; hence the maximum over $\alpha$ should be replaced by the supremum. There remains a possible dependence upon $\tau$. It is not hard to show that $\hat{q}(\tau)$ will be invariant under translations of $\tau$, and hence one might hope to use the ergodic theorem (see [13]) to show that $\hat{q}$ is constant almost everywhere. However, this argument requires that $\hat{q}$ be a measurable function of $\tau$-and while we think this is likely to be true, we do not have a proof.

\section{Appendix A. Proof of Lemma}

Let $\Lambda_{1}$ be a particular rectangular box and let $\Lambda$ be any larger box which can be exactly covered by $\Lambda_{1}$ and its translates, labelled $\Lambda_{j}$, arranged so that no two of these have any sites in common. It then follows from the definition (12) that

$$
R_{\Lambda}(\rho)=\frac{1}{n} \sum_{j=1}^{n} R_{\Lambda_{j}}(\rho),
$$

and thus, from (13), that

$$
Q_{\Lambda}(\lambda, \tau) \leqq \frac{1}{n} \sum_{j=1}^{n} Q_{\Lambda_{j}}(\lambda, \tau),
$$

and therefore, assuming the integrals exist,

$$
\int Q_{\Lambda}(\lambda, \tau) d \mu(\tau) \leqq \int Q_{\Lambda_{1}}(\lambda, \tau) d \mu(\tau)
$$

where we have used the fact that the measure $\mu(\tau)$ is invariant under translations of the lattice, which implies that the integral of $Q_{\Lambda_{j}}$ is independent of $j$. The existence of 
the integrals, given that $\mu$ is a probability measure and $Q_{A}$ is bounded, is assured if $Q_{A}$ is a measurable function of $\tau$ (see Appendix B).

The inequality (A3), and the fact that $Q_{\Lambda} \geqq-1$ shows that $q(\lambda)$ in (14) can be obtained as the limit of a van Hove sequence in which the larger boxes can be exactly covered by copies of the smaller boxes. But if the coverage is not exact, the error is easily bounded, due to the fact that $\rho\left(S_{i}^{(1)} S_{i}^{(2)}\right)$ always lies between -1 and +1 , and this can be used, in a series of classical arguments $[11,14,15]$, to show that the limit is independent of the van Hove sequence.

To establish (15), define

$$
q(\lambda, \tau)=\lim _{\Lambda \rightarrow \infty} \sup Q_{\Lambda}(\lambda, \tau)
$$

for a particular van Hove sequence. It is easy to show that if $t$ is a translation of the lattice, i.e.,

$$
(t \tau)_{i j}=\tau_{i+k, j+k}
$$

for some $k$ in the Bravais lattice, then

$$
q(\lambda, t \tau)=q(\lambda, \tau)
$$

(one way to think about this is to leave $\tau$ fixed and translate the $\Lambda$ ). But then, by the ergodic property [13], $q(\lambda, \tau)$ is constant for almost all $\tau$; integration over $\tau$ then shows that it is the same as $q(\lambda)$.

To show that $q(\lambda)$ is upper semi-continuous, we first show that $Q_{\Lambda}(\lambda, \tau)$ has this property for a fixed $\Lambda$ and $\tau$. Indeed, assume that there were a sequence $\lambda_{j}$ tending to $\lambda_{0}$ such that for some $\varepsilon>0$,

$$
Q_{\Lambda}\left(\lambda_{j}, \tau\right) \geqq Q_{\Lambda}\left(\lambda_{0}, \tau\right)+\varepsilon .
$$

Then from the $\rho_{j}$ which maximize (13) we could pick a subsequence converging (weak-*) to a state $\rho_{0}$ which is an equilibrium state for $\lambda_{0}$, and evidently

$$
R_{\Lambda}\left(\rho_{0}\right) \geqq Q_{\Lambda}\left(\lambda_{0}, \tau\right)+\varepsilon,
$$

which contradicts the definition (13). Thus $Q_{\Lambda}(\lambda, \tau)$ is upper semicontinuous in $\lambda$. Consequently the integral in (14) for a fixed $\Lambda$ is upper semi-continuous in $\lambda$. The corresponding property for $q(\lambda)$ is a consequence of $(14)$ and the fact that the integral for a fixed $\Lambda$ is not less than $q(\lambda)$.

\section{Appendix B. Measurability of $Q_{\Lambda}(\lambda, \tau)$}

Let $\lambda$ and $\Lambda$ be fixed, and let $M$ be some set of sites which includes $\Lambda$. Then it is the case that

$$
Q_{\Lambda}(\lambda, \tau)=\lim _{M \rightarrow \infty} \max _{\mu} R_{\Lambda}\left(\bar{\rho}_{M, \mu}(\lambda, \tau)\right)
$$

where the maximum is over the set of boundary conditions for the region $M$, and the limit is over a van Hove sequence. To see that this is correct, note that any equilibrium state of the infinite system restricted to $\Lambda$ is a convex combination of the 
$\bar{\rho}_{M, \mu}$ states restricted to $\Lambda$, so the lim inf of the right side of (B.1) cannot be less than the left side. On the other hand, if the lim sup were larger than the left side, we could find a subsequence of the van Hove sequence such that the corresponding sequence of states $\bar{\rho}_{M, \mu}$ converged to a state (necessarily an equilibrium state) as $M \rightarrow \infty$ yielding a value for $R_{\Lambda}$ larger than $Q_{A}$ - which is obviously impossible.

For a given $M, \bar{\rho}_{M, \mu}$ is a continuous function of a finite number of $\tau_{i j}$, those with $i$ and $j$ in $\bar{M}$, and is independent of the other $\tau_{i j}$. Thus it is a continuous function of $\tau$, as is the maximum over $\mu$ of $R_{\Lambda}$ on the right side of (B.1). Thus $Q_{\Lambda}$ is a pointwise limit of continuous functions, and hence [16] measurable.

Acknowledgement. The authors are grateful for the hospitality accorded them by the Institut des Hautes Etudes Scientifiques. They wish to thank G. Parisi and D. Ruelle for useful discussions and D. Sherrington for comments on the literature. Research support from the Deutsche Forschungsgemeinschaft (SFB 123) at the University of Heidelberg, and from the U.S. National Science Foundation grant DMR 8108310 at Carnegie-Mellon University is also gratefully acknowledged.

\section{References}

1. Edwards, S. F., Anderson, P. W.: Theory of spin glasses. J. Phys. F5, 965-973 (1975)

2. Blandin, A., Gabay, M., Garel, T. On the mean-field theory of spin glasses. J. Phys. C13, 403-418 (1980)

3. Griffiths, R. B., Lebowitz, J. L.: Random spin systems: Some rigorous results. J. Math. Phys. 9, 1284$92(1968)$

4. Vuillermot, P. A.: Thermodynamics of quenched random spin systems, and application to the problem of phase transitions in magnetic (spin) glasses. J. Phys. A10, 1319-33 (1977)

5. Ledrappier, F.: Pressure and variational principle for random Ising model. Commun. Math. Phys. 56, 297-302 (1977)

6. van Hemmen, J. L., Palmer, R. G.: The thermodynamic limit and the replica method for short range systems. J. Phys. A15, 3881-90 (1983)

7. a. Dobrushin, R. L.: The description of a random field by means of conditional probabilities and conditions of its regularity. Theory Probab. Its. Appl. (USSR) 13, 197-224 (1968)

b. Lanford, O. E. III, Ruelle, D.: Observables at infinity and states with short range correlations in statistical mechanics. Commun. Math. Phys. 13, 194-215 (1969)

8. a. Sewell, G. L.: Statistical mechanical theory of metastable states. Lett. Nuovo Cimento 10, 430-34 (1974)

b. Sewell, G. L.: Stability, equilibrium and metastability in statistical mechanics. Phys. Rep. 57, 307342 (1980)

9. Griffiths, R. B.: Spontaneous magnetization in idealized ferromagnets. Phys. Rev. 152, 240-246 (1966)

10. van Enter, A. C. D., van Hemmen, J. L.: On the nature of the spin glass phase. Preprint, Heidelberg University, 1982

11. Israel, R. B.: Convexity in the theory of lattice gases. Princeton, NJ: Princeton University Press 1979

12. Roos, H.: Statistical mechanics of quantum lattice systems without translational invariance. Commun. Math. Phys. 42, 83-100 (1975)

13. Walters, P.: Ergodic theory. In: Lecture Notes in Mathematics, Vol. 458. Berlin, Heidelberg, New York: Springer 1975

14. Ruelle, D.: Statistical mechanics. Amsterdam, New York: Benjamin 1969

15. Griffiths, R. B.: A proof that the free energy of a spin system is extensive. J. Math. Phys. 5, 1215-22 (1964)

16. Reed, M., Simon, B.: Functional analysis. New York, London: Academic Press 1972, 116

17. Bidaux, R., Carton, J. P., Sarma, G.: (unpublished results) (1977)

18. Sherrington, D., Kirkpatrick, S.: Solvable model of a spin glass. Phys. Rev. Lett. 35, 1792-96 (1975) 
19. Kirkpatrick, S., Sherrington, D.: Infinite-ranged models of spin glasses. Phys. Rev. B17, 4384-4403 (1978)

20. a. Parisi, G.: An introduction to the statistical mechanics of amorphous systems. Les Houches lectures (1982)

b. Parisi, G.: Mean field theory for spin glasses. In: Disordered systems and localization. Lecture Notes in Physics Vol. 149, Castellani, C., Di Castro, C., Peliti, L. (eds.) Berlin, Heidelberg, New York: Springer 1981, pp. 107-117.

Communicated by J. Fröhlich

Received February 7, 1983; in revised form April 19, 1983 
